\title{
ON THE CANONICAL DEGREES OF CURVES IN VARIETIES OF GENERAL TYPE
}

\author{
PASCAL AUTISSIER, ANTOINE CHAMBERT-LOIR, AND CARLO GASBARRI
}

\section{INTRODUCTION}

In this paper, we work in the framework of complex analytic varieties; without contrary mention, varieties are assumed to be irreducible (and reduced). If $C$ is a projective curve, we let $g_{C}$ be its geometric genus (namely, the genus of its desingularization) and $\chi(C)=2-2 g_{C}$ its geometric Euler characteristic; we also write $\operatorname{deg}_{C} L$ for the degree of a line bundle $L$ on $C$.

Given a smooth projective variety of general type $X$ and a integer $g$, a widely believed conjecture predicts that there exists a strict closed subset $Z$ of $X$ and an integer $d$ such that every curve of genus $g$ drawn on $X$ and not contained in $Z$ has degree at most $d$. An effective version of this conjecture can be stated in the following way:

Conjecture 1. Let $X$ be a smooth projective variety of general type; let $K_{X}$ be the canonical line bundle of $X$. Then there exist real numbers $A$ and $B$, and a strict Zariski closed subset $Z \subset X$ such that for every curve $C \subset X$ such that $C \not \subset Z$, we have

$$
\operatorname{deg}_{C}\left(K_{X}\right) \leq A\left(2 g_{C}-2\right)+B .
$$

Combined with the Riemann-Hurwitz formula, this conjecture would imply that for any finite morphism $g: C \rightarrow X$ such that $g(C) \not \subset Z$,

$$
\operatorname{deg}_{C}\left(g^{*} K_{X}\right) \leq A\left(2 g_{C}-2\right)+B[C: g(C)] .
$$

The conjecture is obvious when $\operatorname{dim}(X)=1$. For surfaces, the most important result in its direction is a now well-known theorem by Bogomolov [4] (and its further developments) who proves such a statement under the additional assumption that $X$ is a minimal surface with positive Segre class. Under the supplementary assumption that $X$ is Brody-hyperbolic, i.e., that any holomorphic map from $\mathbf{C}$ to $X$ is constant, Demailly established this conjecture in [8] (Theorem 2.1); he shows that one may take $Z=\emptyset$ and $B=0$.

Of course, an effective geometric upper-bound of the real numbers $A$ and $B$ of the conjecture would be of the utmost interest. In his interesting paper [13. Miyaoka establishes a fundamental inequality between the selfintersection of a projective curve $C$ drawn on a minimal projective surface of general type $X$, its canonical degree and numerical invariants of the surface itself. When one restricts oneself to smooth curves, one has $C^{2}=2 g_{C}-2-$ $\operatorname{deg}_{C}\left(K_{X}\right)$, and Miyaoka can show effectively, for any positive real number $\varepsilon$, the existence of a real number $B$ (depending on $\varepsilon$ ) such that every smooth 
projective curve $C$ contained in $X$ satisfies

$$
\operatorname{deg}_{C} K_{X} \leq\left(\frac{3}{2}+\varepsilon\right)\left(2 g_{C}-2\right)+B .
$$

This analysis can be extended to nodal curves with a bounded number of singular points.

While no method is known to understand the constant $B$, many interesting conjectures and guesses have been made concerning the constant $A$ and its geometric meaning.

Exploiting the analogy between number theory, Nevanlinna theory and algebraic geometry, P. Vojta [17] proposed very deep and ambitious conjectures bounding the canonical height of algebraic points of bounded degree in fibrations. The first version of his conjectures suggests that for any positive real number $\varepsilon$, conjecture 1 could hold with $A=\operatorname{dim}(X)+\varepsilon$, the constant $B$ and the exceptional locus $Z$ depending on $\varepsilon$, provided one restricts oneself to curves of given gonality. (Recall that the gonality of a projective curve is the least degree of a non-constant morphism to the projective line; obviously, horizontal curves of bounded degree in a fibration have bounded gonality.) A more recent version of his conjecture, published in [18] replaces $A=\operatorname{dim} X+\varepsilon$ by the more optimistic $A=1+\varepsilon$, again with the same restriction on gonality.

More prudently, McQuillan [11] asked whether the constant $A$ could be related to the holomorphic sectional curvature of the variety $X$. The holomorphic sectional curvature of hermitian symmetric domains has been explicitly bounded by Azukawa in 2, see Lemma 4 below. Our first contribution in this paper is the observation that this result implies a precise and explicit form of conjecture 1 for compact locally symmetric varieties, with $A=\operatorname{dim}(X)$.

Proposition 2. Let $M$ be a bounded hermitian domain and let $X$ be a compact quotient of $M$. Then, for any projective curve $C$ and any finite morphism $g: C \rightarrow X$,

$$
\operatorname{deg}_{C}\left(g^{*} K_{X}\right) \leq \operatorname{dim}(X)\left(2 g_{C}-2\right) .
$$

Conversely, and this was the original motivation of this note, we also want to indicate, by very elementary examples, that one cannot go under $A=\operatorname{dim}(X)$ in general, or, in other words, that Vojta's refined expectation cannot hold without the above-mentioned restriction on the gonality. Namely, we shall prove the following theorem.

Theorem 3. Let $d$ be an integer such that $d \geq 2$. There exists a smooth projective variety $X$ of dimension d whose canonical bundle $K_{X}$ is ample and a family $\left(C_{n}, f_{n}\right)$, where $C_{n}$ is a smooth projective curve and $f_{n}: C_{n} \rightarrow X$ is a finite morphism satisfying the following properties:

a) $\operatorname{deg}_{C_{n}}\left(f_{n}^{*} K_{X}\right)=d\left(2 g_{C_{n}}-2\right)$;

b) $\lim _{n} g_{C_{n}}=+\infty$;

c) the union of their images $f_{n}\left(C_{n}\right)$ is dense in $X$ for the Zariski topology. 
We show (Theorem 5) that one may take for $X$ any compact Shimura variety associated to any $\mathbf{Q}$-anisotropic, semisimple, simply connected $\mathbf{Q}$ group $\mathbf{G}$, provided $X$ contains at least one totally geodesic compact Riemann surface $C$. The curves $C_{n}$ are then images of $C$ under Hecke correspondences. Using weak approximation at the real place, we show indeed that these curves are Zariski dense in $X$.

We give in a final section an entirely algebraic approach to this Theorem, using powers of Shimura curves. Because of the cusps, considering modular curves in a power of a classical modular curves leads to a slightly weaker result.

We view these examples as a nice implementation of McQuillan's principle that Shimura varieties are a rich source of examples for Diophantine geometry.

A first observation to make is that Theorem 3 does not contradict Miyaoka's result [13] mentioned above. Indeed, there is no reason that the curves $f_{n}\left(C_{n}\right)$ in $X$ are smooth. However, in our examples, the morphism $f_{n}$ will be an immersion in the sense of differential geometry, i.e., its tangent map is injective at each point.

Observe also that Theorem 3 does not contradict Vojta's expectations either, since he restricts himself to curves in a fibration which have bounded degree. The gonality of such curves is obviously bounded, while, according to a theorem of Abramovich [1], the gonality of modular curves tends to infinity.

The geometric conjecture 1 has an arithmetic analogue, due to Vojta. In a similar spirit, we refer to the paper [10] for examples that show that Vojta's conjecture cannot be extended to algebraic points.

Acknowledgments: This work began during an instructional conference on Diophantine Geometry, organized by two of us in University of Rennes under the auspices of Centre Émile Borel and Institut Henri Poincaré. We thank these organizations to have given us the opportunity to discuss this subject.

We also thank B. Edixhoven, A. Levin, M. McQuillan, E. Ullmo and P. Vojta for their interest and their comments.

A.C-L. acknowledges the support of Institut universitaire de France, as well as that of the National Science Foundation under agreement No. DMS0635607 during his stay at the Institute of Advanced Study (Princeton), where part of the work presented here has been done. The three authors are supported by the project Positive of Agence nationale de la recherche, under agreement ANR-2010-BLAN-0119-01.

\section{Hermitian symmetric spaces; Bergman metric}

We first review well known general facts of differential geometry in Hermitian symmetric spaces. Since different authors use different normalizations, we found necessary to recall the precise definitions.

Let $M$ be a Hermitian symmetric space, namely a connected Kähler complex manifold such that each point of $M$ is an isolated fixed point of an involutive holomorphic isometry of $M$. Set $m=\operatorname{dim}(M)$. 
Following the notation of [9] (chap. VIII, $\S 4$ ), let $A(M)$ be the group of holomorphic isometries of $M$ and let $G=A_{0}(M)$ be its identity component; this is a real connected Lie group. We fix a point $o \in M$ and consider its stabilizer $K$ in $A_{0}(M)$; this is a compact subgroup; as a real manifold, $M$ can be identified to the homogeneous space $G / K$.

We shall always assume that $M$ is of noncompact type: $G$ is semisimple and $K$ is a maximal compact subgroup of $G$; then $M$ is simply connected ([9], p. 376, Theorem 4.6) and there exists (9], p. 382, Theorem 7.1) a holomorphic diffeomorphism from $M$ to a bounded symmetric domain $D_{M}$ in the complex space $\mathbf{C}^{m}$, "symmetric" meaning that each point of $D_{M}$ is an isolated fixed point of an involutive holomorphic diffeomorphism of $D_{M}$ onto itself.

Over the tangent bundle $T D_{M}$ of $D_{M}$ there exists a Kähler metric $\omega$, for which the following property holds: Let $\|\cdot\|_{K E}$ the induced metric on the canonical bundle $K_{D_{M}}=\bigwedge^{m} \Omega_{D_{M}}^{1}$, then the following formula relates the Kähler form $\omega$ and the first Chern form of $K_{D_{M}}$ :

$$
\omega=c_{1}\left(K_{D_{M}},\|\cdot\|_{\mathrm{KE}}\right) .
$$

Such a metric is called Kähler-Einstein and in this case it coincides with the Bergman metric. Its existence is proved for instance in Proposition 3.6 (p. 371) of 9]. Once we fix such a normalization, as we do now, this metric is uniquely determined. Any holomorphic diffeomorphism of $D_{M}$ is an isometry with respect to that metric (9, p. 370, Prop. 3.5). We assume, as we may, that the Kähler structure of $M$ is the pull-back of the fixed KählerEinstein metric on $D_{M}$ by some (hence, any) holomorphic diffeomorphism of $M$ to $D_{M}$.

\section{Holomorphic SeCtional CuRVAture}

Let us recall a definition of the holomorphic sectional curvature. Let $p \in M$ and let us consider holomorphic maps $\gamma: U \rightarrow M$, where $U$ is the open unit disk in $\mathbf{C}$, such that $\gamma(0)=0$ and $\gamma^{\prime}(0) \neq 0$. Let us write $\gamma^{*} \omega=F_{\gamma}(z) i \mathrm{~d} z \wedge \mathrm{d} \bar{z}$ on $U$, where $F_{\gamma}$ is nonnegative, $F_{\gamma}(0) \neq 0$. In a neighborhood of 0 , the form $\gamma^{*} \omega$ defines a metric on the canonical bundle $K_{U}$ and its curvature form is given by

$$
R_{\gamma}=\mathrm{dd}^{\mathrm{c}} \log \left(F_{\gamma}\right) .
$$

The Ricci curvature $\operatorname{Ric}_{\gamma}$ of $\gamma^{*} \omega$ is defined by the formula

$$
\operatorname{Ric}_{\gamma}(z) F_{\gamma}(z) i \mathrm{~d} z \wedge \mathrm{d} \bar{z}=-\mathrm{dd}^{\mathrm{c}} \log \left(F_{\gamma}\right)
$$

where $\mathrm{dd}^{\mathrm{c}}=\frac{i}{2 \pi} \partial \bar{\partial}$. This is a function on a neighborhood of 0 in $U$, and its value at $z=0$ only depends on the tangent line at $p$ generated by $\gamma^{\prime}(0)$.

The holomorphic sectional curvature $S_{M}(p)$ at $p$ is then defined as

$$
S_{M}(p)=\sup _{\substack{\gamma: U \rightarrow M \\ \gamma(0)=p}} \operatorname{Ric}_{\gamma}(0)
$$

Since the group of holomorphic isometries of $M$ acts transitively on $M$, the holomorphic sectional curvature is actually independent of $p$; we shall 
denote it by $S_{M}$. Since $M$ is a Hermitian symmetric space of the noncompact type (9], Ch. VIII, Theorem 7.1, p. 382), we know that $S_{M}$ is negative (9], Ch. V, Theorem 3.1, p. 241).

Corollary 8.5 (i) in [2] asserts that the holomorphic sectional curvature satisfies the following inequality:

Lemma 4 (Azukawa). One has

$$
\frac{-1}{S_{M}} \leq \operatorname{dim}(M)
$$

\section{Compact quotients}

Let $\Gamma$ be a cocompact discrete subgroup of $G$ which acts without fixed points on $M$. Then, $X_{\Gamma}=\Gamma \backslash M$ is a compact connected complex manifold. The Kähler-Einstein structure descends to a Kähler-Einstein metric $\omega_{\Gamma}$ on $X_{\Gamma}$. The canonical line bundle $K_{X_{\Gamma}}$ is naturally metrized; in view of Equation (1), its Chern form satisfies

$$
\omega_{\Gamma}=c_{1}\left(K_{X_{\Gamma}},\|\cdot\|\right),
$$

hence is positive. By Kodaira's theorem, $X_{\Gamma}$ is a projective complex manifold.

\section{Compact Riemann surfaces}

Let $Y$ be a smooth compact Riemann surface and let $f: Y \rightarrow X_{\Gamma}$ be a finite morphism. One has

$$
\int_{Y} f^{*}\left(\omega_{\Gamma}\right)=\operatorname{deg}_{Y}\left(f^{*} K_{X_{\Gamma}}\right) .
$$

Moreover, the form $f^{*}\left(\omega_{\Gamma}\right)$ induces a hermitian metric on the canonical line bundle $K_{Y}\left(-\Delta_{f}\right)$ of $Y$, where $\Delta_{f}$ is the branching divisor of $f$. Let $R_{Y}$ be its curvature, one has

$$
\int_{Y} R_{Y}=\operatorname{deg}\left(K_{Y}-\Delta_{f}\right)=-\chi(Y)-\operatorname{deg}\left(\Delta_{f}\right) .
$$

By the definition of the holomorphic sectional curvature,

$$
R_{Y} \geq-S_{M} f^{*}\left(\omega_{\Gamma}\right) .
$$

This shows that

$$
-\chi(Y) \geq-\chi(Y)-\operatorname{deg}\left(\Delta_{f}\right) \geq-S_{M} \operatorname{deg}_{Y}\left(f^{*} K_{X_{\Gamma}}\right) .
$$

Given Lemma 4, this establishes Proposition 2 .

\section{Totally geodesic Riemann surfaces}

Let $(X, \omega)$ be a Kähler complex manifold, let $Y$ be a Riemann surface and let $f: Y \rightarrow X$ be a holomorphic immersion. We say that $f$ is totally geodesic if any geodesic of $X$ contained in $Y$ is a geodesic of $Y$ with respect to the Kähler form $f^{*} \omega$. This is equivalent to the vanishing of the second fundamental form of $Y$ ([9], Theorem 14.5, p. 80) and implies that for each $p \in Y$, the Ricci curvature of $f^{*} \omega$ at $p$ equals the holomorphic sectional curvature of $X$ at $f(p)$. (This follows, for example, from the Gauss equation relating 
the curvature tensor of a Riemannian manifold, that of a submanifold and the second fundamental form, see [3], Theorem 1.72.)

Let us assume that $X=X_{\Gamma}=\Gamma \backslash M$. Any immersion $f: Y \rightarrow X$ lifts to a holomorphic immersion $\tilde{f}: \mathbf{D} \rightarrow M$, where $\mathbf{D}$ is the open unit disk. The conditions for $f$ and $\tilde{f}$ to be totally geodesic are equivalent. Moreover, since automorphisms of $M$ preserve its Kähler form, we obtain that for any such automorphism $g, g \circ \tilde{f}$ is totally geodesic if and only if $\tilde{f}$ is.

For a totally geodesic map $f: Y \rightarrow X_{\Gamma}$, where $Y$ is a compact Riemann surface, inequality (4) becomes an equality:

$$
-\chi(Y)=-S_{M} \operatorname{deg}_{Y}\left(f^{*} K_{X_{\Gamma}}\right) \quad(Y \text { totally geodesic }) .
$$

\section{Constructing one totally geodesic Riemann surface}

It does not seem easy to construct totally geodesic compact Riemann surfaces in arbitrary compact quotients of symmetric hermitian domains. However, let us show some examples, coming from the theory of Shimura varieties.

We restrict the situation to the case where the Lie group $G$ is given by $G=\mathbf{G}(\mathbf{R})$, for some connected semisimple and simply connected $\mathbf{Q}$ anisotropic linear algebraic group $\mathbf{G}$ over $\mathbf{Q}$. Then, for any congruence subgroup $\Gamma$ which acts without fixed point on $M=G / K$, the quotient space $X_{\Gamma}=\Gamma \backslash M$ is a smooth projective variety.

Let $D_{1}$ be a quaternion algebra over $\mathbf{Q}$ and let $\mathbf{G}_{1}=\operatorname{SL}\left(D_{1}\right)$ be the corresponding almost simple $\mathbf{Q}$-group. We assume that $D_{1}$ is not split, i.e., is not isomorphic to $\mathrm{M}_{2}(\mathbf{Q})$ but that $D_{1} \otimes_{\mathbf{Q}} \mathbf{R}$ is split, so that $G_{1}=\mathbf{G}_{1}(\mathbf{R}) \simeq$ $\mathrm{SL}_{2}(\mathbf{R})$. Let $K_{1} \simeq \mathrm{SO}_{2}(\mathbf{R})$ be a maximal compact subgroup in $G_{1}$, let $M_{1}=G_{1} / K_{1}$; then $M_{1}$ is a hermitian symmetric domain, and is isomorphic to the Poincaré upper half-plane. Finally, let $\Gamma_{1}$ be a congruence subgroup in $G_{1}(\mathbf{Q})$, small enough so as to acts freely on $M_{1}$. Then $X_{1}=\Gamma_{1} \backslash M_{1}$ is a smooth curve.

Let $F$ be a totally real field such that the simple $F$-algebra $D=D_{1} \otimes_{\mathbf{Q}} F$ is not split. Let $\mathbf{G}=\mathrm{SL}(D)$ be the corresponding almost simple $F$-group; by Weil's restriction of scalars, we view it as a semisimple $\mathbf{Q}$-group. One has $G=\mathbf{G}(\mathbf{R}) \simeq \mathrm{SL}(2, \mathbf{R})^{d}$, where $d=[F: \mathbf{Q}]$. Similarly, let $K$ be a maximal compact subgroup of $G$, let $M=G / K$ and let $\Gamma$ be a congruence subgroup of $\mathbf{G}(\mathbf{Q})$ acting freely on $M$. Then, $X=\Gamma \backslash M$ is a smooth projective variety.

Moreover, the algebraic group $\mathbf{G}_{1}$ embeds naturally in $\mathbf{G}$; over $\mathbf{R}$, this is the diagonal embedding $\mathrm{SL}(2, \mathbf{R}) \hookrightarrow \mathrm{SL}(2, \mathbf{R})^{d}$. Let us assume that $K$ contains $K_{1}$ and that $\Gamma$ contains $\Gamma_{1}$. Then, the immersion $\mathbf{G}_{1} \hookrightarrow \mathbf{G}$ induces an immersion $X_{1} \hookrightarrow X$ whose image is a totally geodesic Riemann surface. (This follows, from example, from [9], Theorem 7.2, p. 224. See also [14], §4.1.)

Let us finally remark that in this example, $M$ is a power of the Poincaré disk, hence its holomorphic sectional curvature is equal to $-1 / \operatorname{dim}(M)$.

\section{HECKE OPERATORS}

Any $g \in G(\mathbf{Q})$ gives rise to a Hecke correspondence $T_{g}$ on $X_{\Gamma}$. Let us recall its definition. Let $\Gamma_{g}=\Gamma \cap g^{-1} \Gamma g$; this is a congruence subgroup of 
finite index in $\Gamma$. The variety $\Gamma_{g} \backslash M$ is smooth and admits two étale maps to $X_{\Gamma}$, given respectively by

$$
p_{1}\left(\Gamma_{g} x\right)=\Gamma x, \quad p_{2}\left(\Gamma_{g} x\right)=\Gamma g x,
$$

for any $x \in M$, hence a correspondence on $X_{\Gamma}$.

Let us describe explicitly the image of a subvariety $Y \subset X_{\Gamma}$ by this correspondence. Let $\left(h_{1}, \ldots, h_{r}\right)$ be a family of elements of $\Gamma$ which represent each left-class modulo $\Gamma_{g}$. Let $\tilde{Y} \subset M$ be the preimage of $Y$; then,

$$
T_{g}([Y])=\sum_{i=1}^{r}\left[p\left(g h_{i} \tilde{Y}\right)\right]
$$

where $p: M \rightarrow X_{G}$ is the natural projection.

In view of this description, we observe that if $Y$ is the totally geodesic image in $X_{\Gamma}$ of a Riemann surface, then so are all the irreducible components of $T_{g}([Y])$.

\section{WEAK APPROXIMATION}

Since the group $G$ is connected, $\mathbf{G}(\mathbf{Q})$ is dense in $G=\mathbf{G}(\mathbf{R})$ (15], Theorem 7.7, p. 415). Let $\tilde{Y} \subset G$ be the preimage of $Y$ by the projection map $G \rightarrow X_{\Gamma}=\Gamma \backslash G / K$. The union of the curves $a \tilde{Y}$, for $a \in G(\mathbf{Q})$, is therefore dense in $G$. It follows that the union of the curves $T_{a} Y$ is dense in $X_{\Gamma}$.

Let $Y_{0}$ be any fixed totally geodesic Riemann surface in $X_{\Gamma}$. We conclude that the irreducible components of the curves $T_{a} Y_{0}$, for $a \in G(\mathbf{Q})$, are dense in $X_{\Gamma}$. Since these components are also totally geodesic, we obtain the following result.

Theorem 5. Let $X_{\Gamma}$ be a compact Shimura variety associated to a semisimple, connected and simply connected $\mathbf{Q}$-anisotropic algebraic group over $\mathbf{Q}$; let us assume that $X_{\Gamma}$ contains a totally geodesic Riemann surface.

Then, the set of projective (algebraic) curves $Y$ in $X_{\Gamma}$ such that

$$
\operatorname{deg}_{K_{X_{\Gamma}}}(Y)=\left(\frac{-1}{S_{X_{\Gamma}}}\right)\left(2 g_{Y}-2\right)
$$

is dense in $X_{\Gamma}$ for the complex topology; in particular, it is Zariski dense.

Remark 6. When it applies, a theorem of Clozel, Oh and Ullmo [7 refines the density of Hecke orbits into an equidistribution theorem.

\section{SHIMURA CURVES}

In this section we give another, more explicit, approach to Theorem 3 , It is obtained by considering products of Shimura curves and Hecke correspondences over them. Strictly speaking this section can be viewed as a particular case of the previous one but the present approach is entirely algebraic and relies on the modular interpretation of the Shimura curves. A similar analysis can be applied to the graph of Hecke correspondences on modular curves; because of the cusps, this leads to a slightly weaker form of Theorem 3 ,

Our main references concerning Shimura curves and their moduli interpretation are [12, 6]; see also [16, 5]. 
Let $D$ be an indefinite quaternion algebra over $\mathbf{Q}$ of reduced discriminant $\delta>1$, viewed as a subalgebra of $\mathrm{M}_{2}(\mathbf{R})$; let $R$ be a fixed maximal order in $D$ (they are all conjugate) and let $\Gamma_{R}$ be the subgroup $R^{*} \cap \mathrm{SL}_{2}(\mathbf{R})$ of $\mathrm{GL}_{2}(\mathbf{R})$. Elements of $\Gamma_{R}$ act by homographies on the Poincaré upperhalf-plane $\mathfrak{h}$. The Shimura curve $C$ associated to $D$ is the quotient of $\mathfrak{h}$ by $\Gamma_{R}$. It is a compact algebraic complex curve whose points are in one-toone correspondence with the set of (isomorphism classes of) complex abelian surfaces endowed with an action of $R$, also called false elliptic curves. These abelian surfaces carry a canonical principal polarization once an element of square $-\delta$ is fixed in $R$.

See also [6, p. 594] for an adelic description of this Shimura curve, in the spirit of the general theory of Shimura varieties, as well as for its coverings induced by level-structures on these abelian surfaces. In particular, for any square free positive integer $N$ which is prime to $\delta$, there exists a projective curve $C_{N}$ parameterizing isogenies of false degree $N$ between false elliptic curves, that is isogenies $f: A \rightarrow A^{\prime}$ commuting with the action of $R$ such that the composition $f^{\vee} \circ f$ of $f$ with its dual isogeny $f^{\vee}$ is the multiplication by $N$. There are natural maps $s_{M}^{N}: C_{N} \rightarrow C_{M}$ and $t_{M}^{N}: C_{N} \rightarrow C_{M}$ between these curves, whenever $M$ divides $N$. If $N \geq 4$, this curve is a fine moduli space; equivalently, $C_{N}(\mathbf{C})$ is the quotient of the upper-half-plane by a discrete, cocompact, and torsion free subgroup of $\mathrm{SL}_{2}(\mathbf{R})$. Since there are no cusps at infinity, the complex description of these curves, or their moduli interpretation, shows that these maps are finite and étale.

Let $p>4$ be a fixed prime number, let $d \geq 2$ be a positive integer and let $X$ be the smooth variety $C_{p}^{d}$. Let $N=p \ell_{1} \ldots \ell_{d-1}$ be the product of $d$ distinct prime numbers not dividing $\delta$, among which the prime $p$. The curve $C_{N}$ admits $d$ distinct morphisms $g_{1}, \ldots, g_{d}$ to the curve $C_{p}$, given by $g_{d}=s_{p}^{N}$ and $g_{i}=t_{p}^{p \ell_{i}} \circ s_{p \ell_{i}}^{N}$ for $1 \leq i \leq d-1$. Let $f_{N}: C_{N} \rightarrow X$ be the morphism $\left(g_{1}, \ldots, g_{d}\right)$.

Our claim is that the variety $X$ and the curves $\left(C_{N}, f_{N}\right)$ we obtain satisfy the statement of Theorem 3 . Since one has

$$
\operatorname{deg} f_{N}^{*} K_{X}=-d \chi\left(C_{N}\right)
$$

for any integer $N$, this follows from the following density property.

Lemma 7. Let $p$ and $d$ be fixed. Then the union of the curves $f_{N}\left(C_{N}\right)$ is dense for the Zariski topology, where $N$ varies among the set of integers of the form $N=\ell_{1} \ldots \ell_{d-1} p$ as above.

Proof. The proof is by induction on $d$. When $d=1$, it clearly holds since $X=C_{p}$ and $f_{p}: C_{p} \rightarrow X$ is the identity map. Let us assume that the result holds for $d-1$ and let us prove it for $d$. Assume by contradiction that there exists a divisor $H$ in $X$ containing the images of all curves $C_{N}$ above.

Let $X^{\prime}=C_{p}^{d-1}$ and let $q: X \rightarrow X^{\prime},\left(x_{1}, \ldots, x_{d}\right) \mapsto\left(x_{2}, \ldots, x_{d}\right)$, be the projection obtained by forgetting the first component. For any integer $N^{\prime}$ of the form $p \ell_{2} \ldots \ell_{d-1}$ which is the product of distinct prime numbers, let $f_{N^{\prime}}^{\prime}: C_{N^{\prime}} \rightarrow X^{\prime}$ be the corresponding morphism. If $\ell_{1}$ is any prime number 
not dividing $N^{\prime}$ and $N=\ell_{1} N^{\prime}$, there is a commutative diagram

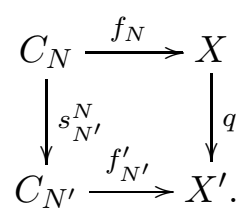

By hypothesis, all curves $f_{N^{\prime}}^{\prime}\left(C_{N^{\prime}}\right)$ are contained in $q(H)$, hence $q(H)=X^{\prime}$ by induction. Since $\operatorname{dim} H=\operatorname{dim} X^{\prime}$ and the restriction $q_{H}$ of $q$ to $H$ is surjective, there exists a point $y^{\prime} \in C_{N^{\prime}}$ such that $q_{H}$ is finite above $x^{\prime}=f_{N^{\prime}}^{\prime}\left(y^{\prime}\right)$. This point $y^{\prime}$ corresponds to an isogeny $u: A \rightarrow A^{\prime}$ between abelian surfaces endowed with an action of $R$, commuting with the action on $R$, such that $u^{\vee} \circ u$ is the multiplication by $N^{\prime}$.

For any prime number $\ell$ which does not divide $N^{\prime}$ and which is totally decomposed in $R$, there exists a cyclic subgroup $K_{\ell}$ of order $\ell$ in $A$ which is invariant by $R$. The subgroup $K_{\ell}+\operatorname{ker}(u)$ of $A$ is then cyclic of order $N=$ $\ell N^{\prime}$, and $R$-invariant, so that the point $f_{N}\left(A \rightarrow A /\left(K_{\ell}+\operatorname{ker}(u)\right)\right)$ of $X$ is a point of $H$ mapping to $f_{N^{\prime}}^{\prime}\left(A \rightarrow A^{\prime}\right)$ by $q$. Then, $\operatorname{pr}_{1}\left(f_{N}\left(A \rightarrow A /\left(K_{\ell}+\right.\right.\right.$ $\operatorname{ker}(u))))=g_{1}\left(A \rightarrow A /\left(K_{\ell}+\operatorname{ker}(u)\right)\right)=\left(A / K_{\ell} \rightarrow A /\left(K_{\ell}+\operatorname{ker}(u)\right)\right)$.

Moreveor, for any given prime number $\ell$ which does not divide $\delta$, there are infinitely many prime numbers $\ell^{\prime}$ such that our false elliptic curves have no isogeny of false degree $\ell \ell^{\prime}$, for, otherwise, almost all prime numbers would be reduced norms in $D$. Consequently, the fibre of $q_{H}$ above $f_{N^{\prime}}^{\prime}\left(A \rightarrow A^{\prime}\right)$ is infinite, contradiction.

\section{REFERENCES}

[1] D. Abramovich - "A linear lower bound on the gonality of modular curves", Internat. Math. Res. Notices (1996), no. 20, p. 1005-1011.

[2] K. Azukawa - "Curvature operator of the Bergman metric on a homogeneous bounded domain", Thoku Math. J. 37 (1985), no. 2, p. 197-223.

[3] A. L. Besse - Einstein manifolds, Classics in Mathematics, Springer-Verlag, Berlin, 2008, Reprint of the 1987 edition.

[4] F. A. Bogomolov - "Families of curves on a surface of general type", Dokl. Akad. Nauk SSSR 236 (1977), no. 5, p. 1041-1044.

[5] J.-F. Boutot \& H. Carayol - "Uniformisation p-adique des courbes de Shimura: les théorèmes de Čerednik et de Drinfel'd", Astérisque, no. 196-197, p. 7, 45-158 (1992), Astérisque 196-197, Soc. Math. France, 1991.

[6] K. BuzzARD - "Integral models of certain Shimura curves", Duke Math. J. 87 (1997), no. 3, p. 591-612.

[7] L. Clozel, H. OH \& E. Ullmo - "Hecke operators and equidistribution of Hecke points", Invent. Math. 144 (2001), no. 2, p. 327-351.

[8] J.-P. Demailly - "Algebraic criteria for Kobayashi hyperbolic projective varieties and jet differentials", in Algebraic geometry-Santa Cruz 1995, Proc. Sympos. Pure Math., vol. 62, Amer. Math. Soc., Providence, RI, 1997, p. 285-360.

[9] S. Helgason - Differential geometry, Lie groups, and symmetric spaces, Graduate Studies in Mathematics, vol. 34, American Mathematical Society, Providence, RI, 2001, Corrected reprint of the 1978 original.

[10] A. LEVIN - "The exceptional set in Vojta's conjecture for algebraic points of bounded degree", 2011, arXiv:1011.6561.

[11] M. MCQuillan - "Non-commutative Mori theory", Preprint IHES/M/01/42, IHES, 2001. 
[12] T. MiYAKe - Modular forms, english éd., Springer Monographs in Mathematics, Springer-Verlag, Berlin, 2006, Translated from the 1976 Japanese original by Yoshitaka Maeda.

[13] Y. MiYAoKA - "The orbibundle Miyaoka-Yau-Sakai inequality and an effective Bogomolov-McQuillan theorem", Publ. RIMS. Kyoto Univ. 44 (2008), no. 2, p. 403417.

[14] B. Moonen - "Linearity properties of Shimura varieties. I", J. Algebraic Geometry 7 (1998), no. 3, p. 539-567.

[15] V. Platonov \& A. RAPinchuk - Algebraic groups and number theory, Pure and Applied Mathematics, vol. 139, Academic Press Inc., Boston, MA, 1994, Translated from the 1991 Russian original by Rachel Rowen.

[16] M.-F. VignÉRAs - Arithmétique des algèbres de quaternions, Lecture Notes in Math., no. 800, Springer-Verlag, 1980.

[17] P. VoJTA - Diophantine approximations and value distribution theory, Lecture Notes in Mathematics, vol. 1239, Springer-Verlag, Berlin, 1987.

[18] _ , "A more general ABC conjecture", Int. Math. Res. Not. 21 (1998), p. 11031116.

imB, Université Bordeaux 1, 351 cours de la Libération, F-33405 Talence Cedex

E-mail address: pascal.autissier@math.u-bordeaux1.fr

Irmar, Université de Rennes 1, Campus de Beaulieu, F-35042 Rennes Cedex, FRANCE

E-mail address: antoine.chambert-loir@univ-rennes1.fr

IRMA, Université de Strasbourg, 7, Rue René Descartes, F-67000 StrasBOURG

E-mail address: gasbarri@math.unistra.fr 